\title{
EXTREME POINTS OF CERTAIN BANACH SPACES RELATED TO CONDITIONAL EXPECTATIONS
}

\author{
JOHN DAUGHTRY \\ Department of Mathematics, East Carolina University, Greenville, NC 27858 \\ e-mail:daughtryjo@mail.ecu.edu \\ and BARNET WEINSTOCK \\ Department of Mathematics, University of North Carolina at Charlotte, Charlotte, NC 28223 \\ e-mail:bmweinst@email.uncc.edu
}

(Received 21 November, 2001; accepted 11 March, 2003)

\begin{abstract}
Let $(X, \mathcal{F}, \mu)$ be a complete probability space and let $\mathcal{B}$ be a sub- $\sigma$ algebra of $\mathcal{F}$. We consider the extreme points of the closed unit ball $\mathbb{B}(\mathcal{A})$ of the normed space $\mathcal{A}$ whose points are the elements of $L^{\infty}(X, \mathcal{F}, \mu)$ with the norm $\|f\|=\|\Phi(|f|)\|_{\infty}$, where $\Phi$ is the probabilistic conditional expectation operator determined by $\mathcal{B}$. No $\mathcal{B}$ measurable function is an extreme point of the closed unit ball of $\mathcal{A}$, and in certain cases there are no extreme points of $\mathbb{B}(\mathcal{A})$.

For an interesting family of examples, depending on a parameter $n$, we characterize the extreme points of the unit ball and show that every element of the open unit ball is a convex combination of extreme points. Although in these examples every element of the open ball of radius $\frac{1}{n}$ can be shown to be a convex combination of at most $2 n$ extreme points by elementary arguments, our proof for the open unit ball requires use of the $\lambda$-function of Aron and Lohman. In the case of the open unit ball, we only obtain estimates for the number of extreme points required in very special cases, e.g. the $\mathcal{B}$-measurable functions, where $2 n$ extreme points suffice.
\end{abstract}

2000 Mathematics Subject Classification. 46B20.

Let $(X, \mathcal{F}, \mu)$ be a complete probability space and let $\mathcal{B}$ be a sub- $\sigma$-algebra of $\mathcal{F}$. For $1 \leq p \leq \infty$ we use the notation $L^{p}(\mathcal{B})$ for $L^{p}\left(X, \mathcal{B},\left.\mu\right|_{\mathcal{B}}\right)$ and henceforth we write $\mu$ in place of $\left.\mu\right|_{\mathcal{B}}$. The norm in $L^{p}(\mathcal{F})$ is denoted as usual by $\|\cdot\|_{p}$. Let $\Phi: L^{1}(\mathcal{F}) \rightarrow L^{1}(\mathcal{B})$ be the conditional expectation operator, so that for $f \in L^{1}(\mathcal{F}), \Phi(f)$ is the unique $\mathcal{B}$-measurable function such that

$$
\int_{B} \Phi(f) d \mu=\int_{B} f d \mu
$$

for all $B \in \mathcal{B}$. We recall that $\Phi$ is a surjective, positive, idempotent operator which satisfies $\Phi(\Phi(f) g)=\Phi(f) \Phi(g)$ for $f, g \in L^{1}(\mathcal{F})$. Let $\mathcal{A}$ be the normed vector space consisting of $L^{\infty}(\mathcal{F})$ with the norm $\|f\|=\|\Phi(|f|)\|_{\infty}$. We study the extreme points of the unit ball $\mathbb{B}(\mathcal{A})$ of $\mathcal{A}$ in several interesting cases.

The motivation for the study of this normed space comes from two sources. In [3] a Banach algebra $\mathcal{D}$ of bounded operators on a $C^{*}$ algebra $\mathfrak{A}$ equipped with a conditional expectation operator $\Phi$ was extensively studied. In certain cases, if $\mathfrak{A}=L^{\infty}(\mathcal{F})$ and $\Phi$ is 
the conditional expectation whose range is $L^{\infty}(\mathcal{B})$ then $\mathcal{D}$ is isometrically isomorphic to $\mathcal{A}$. The other motivation for studying this norm comes from [5], where Lambert studies the set $\mathcal{K}$ of measurable functions $f$ such that $f g \in L^{1}(\mathcal{F})$ for all $g \in L^{1}(\mathcal{B})$. He shows in particular that $L^{\infty}(\mathcal{F}) \subset \mathcal{K} \subset L^{1}(\mathcal{F})$, and that $\mathcal{K}$ is a Banach space with the norm $\|f\|_{\mathcal{K}}=\|\Phi(|f|)\|_{\infty}$. He also proves that $\mathcal{K}=L^{\infty}(\mathcal{F})$ if and only if there exists a constant $C$ such that $|f| \leq C \Phi(|f|)$ a.e., while $\mathcal{K}=L^{1}(\mathcal{F})$ if and only if $\mathcal{B}$ is generated by a finite partition of $X$.

All statements about sets and functions should be understood as ignoring sets of measure 0. All Banach spaces (including the $L^{p}$ spaces) have the complex numbers as the scalar field.

Most of our results deal with one or more of the following examples. For $X \subset \mathbb{R}^{n}$ with finite Lebesgue measure, $\mathcal{L}(X)$ will denote the $\sigma$-algebra of Lebesgue measurable subsets of $X$ and $\mu_{X}$ will denote normalized Lebesgue measure on $X$.

EXAMPLE 1. $X=[-1,1], \mathcal{F}=\mathcal{L}(X), \mu=\mu_{X}$ and $\mathcal{B}$ is the family of sets which are symmetric about 0 . In this case $\Phi(f)(x)=\frac{1}{2}\{f(x)+f(-x)\}$. The functions in the range of $\Phi$ are the even functions, and those in the kernel of $\Phi$ are the odd functions. Also, $|f| \leq 2 \Phi(|f|)$ a.e., so that $\|f\|_{\infty} \leq 2\|f\|$.

EXAmPle 2. $X=[0,1] \times[0,1], \mathcal{F}=\mathcal{L}(X), \mu=\mu_{X}$, and $\mathcal{B}=\{E \times[0,1]: E \in$ $\mathcal{L}([0,1])\}$. Here $\Phi(f)(x, y)=\int_{0}^{1} f(x, t) d t$. The functions in the range of $\Phi$ are those that are independent of the second coordinate.

Example 3. $X=[0,1], \mathcal{F}=\mathcal{L}(X), \mu=\mu_{X}$. Fix $n \in\{2,3,4 \ldots\}$ and let $s:[0,1] \rightarrow$ $[0,1]$ be defined by $s(x)=x+\frac{1}{n}(\bmod 1)$. Let $\mathcal{B}=\left\{E: s^{-1}(E)=E\right\}$. In this case $\Phi(f)(x)=n^{-1} \sum_{j=0}^{n-1} f\left(s^{j}(x)\right)$, where $s^{j}$ denotes the $j$ th iteration of $s$. The functions $f$ in the range of $\Phi$ are those for which the $n$ graphs of $f$ restricted to the intervals $\left[\frac{j-1}{n}, \frac{j}{n}\right], 1 \leq j \leq n$, are all congruent. Also, $|f| \leq n \Phi(|f|)$ a.e. so that $\|f\|_{\infty} \leq n\|f\|$.

EXAmple 4. $X$ is a subset of $\mathbb{R}^{n}$ of finite Lebesgue measure, $\mathcal{F}=\mathcal{L}(X), \mu=\mu_{X}$ and $\mathcal{B}$ is the sub- $\sigma$-algebra of $\mathcal{F}$ generated by a finite partition $\left\{B_{1}, \ldots, B_{n}\right\}$ of $X$ into sets of positive measure. In this case $\Phi(f)=\sum_{i=1}^{n}\left(\frac{1}{\mu\left(B_{i}\right)} \int_{B_{i}} f d \mu\right) \chi_{B_{i}}$.

For convenience we shall use some of the terminology developed in [4] and [2] for describing the structure of sub- $\sigma$-algebras of a measure space.

If $E \subset X$, let $\mathcal{B}_{E}$ denote the relative $\sigma$-algebra $\{E \cap B: B \in \mathcal{B}\}$.

If $E \in \mathcal{F}$, we say that $E$ is a localizing set for $\mathcal{B}$ if $\mu(E)>0$ and $\mathcal{F}_{E}=\mathcal{B}_{E}$. We say that $E \in \mathcal{F}$ is an antilocalizing set for $\mathcal{B}$ if $\mu(E)>0$ and $E$ contains no localizing sets for $\mathcal{B}$. It is proved in [2] that in the case of Example 2 there are no localizing sets for $\mathcal{B}$. It is easy to see that there are no localizing sets in Example 4 either.

A maximal localizing partition is a countable disjoint collection $\left\{E_{i}\right\}_{i \geq 1}$ with $\mu\left(E_{i}\right) \geq \mu\left(E_{i+1}\right)$ for each $i$ such that $E_{1}$ is a maximal localizing set and, if $i>1, E_{i}$ is a maximal localizing set in $X_{i}=X \backslash \cup_{j<i} E_{j}$. It is easy to see that in the case of Example 1, the maximal localizing sets are the sets $E$ such that $E \cap(-E)=\emptyset$ and $E \cup(-E)=X$. In particular, $\{E,-E\}$ is a maximal localizing partition of $[-1,1]$. Similarly in the case of Example 3, each of the intervals $\left[\frac{j-1}{n}, \frac{j}{n}\right]$ is a maximal localizing set, and $\cup_{j}\left[\frac{j-1}{n}, \frac{j}{n}\right]$ is a maximal localizing partition. Indeed, in Example 3, for any maximal localizing set $E$, its successive translates under the mapping $x \rightarrow x+\frac{1}{n}(\bmod 1)$ constitute a maximal localizing partition made up of maximal localizing sets. 
The maximal nondiscriminating set $B_{0}$ is the union of the collection of localizing sets for $\mathcal{B}$ that are $\mathcal{B}$-measurable. It is shown in [6] that functions in the kernel of $\Phi$ vanish on $B_{0}$. In the four examples above, $B_{0}=\emptyset$.

When $\mathfrak{A}=L^{\infty}(\mathcal{F})$, the algebra $\mathcal{D}$ studied in [3] consists of the operators $D_{f}$, $f \in L^{\infty}(\mathcal{F})$, defined by $D_{f}(g)=\Phi(f) g-f \Phi(g)$ for $g \in L^{\infty}(\mathcal{F})$. It is easy to see that $D_{f} D_{h}=D_{\Phi(f) g}$, and it is shown in [3] that $\mathcal{D}$ is closed in the operator norm. The representation $f \rightarrow D_{f}$ is faithful if and only if the maximal nondiscriminating set $B_{0}=\varnothing$, This is the case in each of the examples described above. Note also that $D_{1}$ is always a left identity for $\mathcal{D}$. One can show, at least in the case of Example 1, that the algebra $\mathcal{A}$ is isometric to $\mathcal{D}$. These comments motivate the following proposition.

Proposition 1. $\mathcal{A}$ is a normed algebra when considered with the multiplication $f \times g=\Phi(f) g$.

Proof. Since $\Phi$ is a conditional expectation operator, we have $|\Phi(f) g| \leq \Phi(|f|)|g|$ and hence $\Phi(|\Phi(f) g|) \leq \Phi(\Phi(|f|)|g|)=\Phi(|f|) \Phi(|g|)$.

We begin with some observations about the apparent scarcity of extreme points in the unit ball of $\mathcal{A}$ to contrast with our main results below. The constant function 1 is clearly an element of norm one, and its representation in the algebra $\mathcal{D}$ is a left identity. Thus, by analogy with $\mathrm{C}^{*}$-algebras, the constant function 1 might seem like a good candidate for an extreme point. That idea is demolished by Theorem 1 .

THEOREM 1. Let $e \in \mathbb{B}(\mathcal{A})$ satisfy $\Phi(e)=e$. Then $e$ is not an extreme point of the unit ball.

Proof. Note first that $\|1\|=1$. Let $g$ be a real-valued function such that $\|g\|_{\infty}=\frac{1}{2}$ and $\Phi(g)=0$. Then $1 \pm g \geq 0$ and so $\|1 \pm g\|=\|\Phi(1 \pm g)\|_{\infty}=1$. Hence 1 is not an extreme point of $\mathbb{B}(\mathcal{A})$. Now $e \times(1 \pm g)=e \pm e g$. By Proposition 1, $\|e \pm e g\| \leq\|e\|\|1 \pm g\|=1$. Thus $e$ is not extreme.

Note that the argument in the first part of the proof of Theorem 1 also shows that no positive (or negative) function $f$, which is bounded away from zero, is an extreme point of $\mathbb{B}(\mathcal{A})$.

In special cases one can say more.

THeOREM 2. Let $X, \mathcal{F}, \mathcal{B}, \mu$ be as in Example 1. Let $f \in \mathcal{A}$ be an odd function. Then $f$ is not an extreme point of the unit ball in $\mathcal{A}$.

Proof. Let $g(x)=\frac{f(x)}{|f(x)|}$, where $f(x) \neq 0$ and $x>0, g(x)=\frac{-f(x)}{|f(x)|}$, where $f(x) \neq 0$ and $x<0$, and $g(x)=0$ otherwise. Then $f$ is the average of $f \pm g$.

$$
\|f \pm g\|=\frac{1}{2} \sup _{x}[|g(x) \pm f(x)+| g(-x) \pm f(-x) \mid] .
$$

Assuming that $f$ is in the unit ball of $\mathcal{A}$ and using the fact that $f$ is odd, we see that $|f(x)| \leq 1$ for all $x$. Exclude the case $f=0$, for which the result is obviously true. It follows from the fact that $f$ is odd that

$$
\|f \pm g\|=\frac{1}{2} \sup _{x}|g(x)|[1 \pm|f(x)|+1 \mp|f(x)|]=1 .
$$

THEOREM 3. If $X, \mathcal{F}, \mathcal{B}$, and $\mu$ are as in Example 2 or as in Example 4, there are no extreme points of $\mathbb{B}(\mathcal{A})$. 
Proof. First consider the case of Example 2. Suppose that $\left\|\int_{0}^{1}|f(x, t)| d t\right\|_{\infty}=1$ almost everywhere. Define $w$ by

$$
\begin{aligned}
& w(x)= \\
& \begin{cases}\inf \left\{u: \int_{0}^{u}|f(x, t)| d t=\frac{1}{2} \int_{0}^{1}|f(x, t)| d t\right\}, & \text { if } \exists u \text { with } \int_{0}^{u}|f(x, t)| d t=\frac{1}{2} \int_{0}^{1}|f(x, t)| d t, \\
0, & \text { otherwise. }\end{cases}
\end{aligned}
$$

Let $g(x, y)=-f(x, y) \chi_{[0, w(x)]}(y)+f(x, y) \chi_{[w(x), 1]}(y)$. Then

$$
\begin{aligned}
& f(x, t)+g(x, t)=2 f(x, t) \chi_{[w(x), 1]}(t), \\
& f(x, t)-g(x, t)=2 f(x, t) \chi_{[0, w(x)]}(t),
\end{aligned}
$$

so that

$$
\begin{aligned}
& \int_{0}^{1}|f(x, t)+g(x, t)| d t=2 \int_{w(x)}^{1}|f(x, t)| d t \\
& \int_{0}^{1}|f(x, t)-g(x, t)| d t=2 \int_{0}^{w(x)}|f(x, t)| d t .
\end{aligned}
$$

Thus $\|f \pm g\|=1$, and so $f$ is not an extreme point of $\mathbb{B}(\mathcal{A})$.

Now let $X, \mathcal{F}, \mathcal{B}$, and $\mu$ be as in Example 4. Let $\mu_{i}$ be the measure on $B_{i}$ defined by $\mu_{i}(E)=\mu\left(B_{i}\right)^{-1} \mu(E)$. Then $\mathcal{A}$ is isometrically isomorphic to the direct sum $\bigoplus_{i} L^{\infty}\left(B_{i}\right)$ where each summand is given the norm inherited from $L^{1}\left(B_{i}, \mu_{i}\right)$ and the direct sum is given the norm $\left\|\left(f_{1}, \ldots, f_{n}\right)\right\|=\max _{i}\left\|f_{i}\right\|_{1}$. The desired conclusion now follows easily from the fact that the unit ball of $L^{1}\left(B_{i}\right), 1 \leq i \leq n$, has no extreme points.

Now that we have looked at results which suggest that extreme points for the unit ball in $\mathcal{A}$ are scarce, we wish to consider the opposite side of the coin. We begin with a result about the existence of extreme points in $\mathbb{B}(A)$ in the case of Example 1 and Example 3.

TheORem 4. Let $X, \mathcal{F}, \mathcal{B}, \mu$ be as in Example 1 or Example 3. Let E be a maximal localizing set for $\mathcal{B}$. Let $u$ be a function such that $|u|=1$ on $E$ and $u=0$ otherwise. Then nu is an extreme point of $\mathbb{B}(A)$.

Proof. We give the proof in the context of Example 3. The same proof works with obvious modifications for Example 1. Note first that $\Phi(|n u|)$ is the constant function 1 so that $n u \in \mathbb{B}(A)$. Suppose that $n u=\frac{1}{2}(g+h)$ with $\|g\|,\|h\| \leq 1$. Since $\left.n u\right|_{E}$ is an extreme point of the ball of radius $n$ in $L^{\infty}(E)$ and since $\|g\|_{\infty},\|h\|_{\infty} \leq n$, we have $n u=g=h$ on $E$. Also,

$$
\Phi(n u)=\frac{1}{2}\{\Phi(g)+\Phi(h)\}
$$

and $\|\Phi(g)\|_{\infty} \leq\|g\| \leq 1,\|\Phi(h)\|_{\infty} \leq\|h\| \leq 1$. Since for each $x \in X$ there exist $t \in E$ and $k \in\{1, \ldots, n\}$ with $x=t+\frac{k}{n}(\bmod 1), \Phi(n u)$ is a function of modulus 1 and hence an extreme point of the unit ball of $L^{\infty}([0,1])$. Thus $\Phi(n u)=\Phi(g)=\Phi(h)$, so that

$$
g=n u+k_{1}, h=n u+k_{2},
$$


where $\Phi\left(k_{1}\right)=\Phi\left(k_{2}\right)=0$. But $k_{1}=k_{2}=0$ on $E, n u=0$ on $[0,1] \backslash E$, and $|n u|=n$ on $E$. Since $\|\Phi(|g|)\|_{\infty} \leq 1$ and $\|\Phi(|h|)\|_{\infty} \leq 1$, it follows from the algorithm for $\Phi$ in this case that $k_{1}=k_{2}=0$.

One of the important criteria for whether a unit ball has "enough extreme points" is whether every element of the open unit ball is a convex combination of finitely many extreme points. Our ultimate goal is to establish that in two cases: Example 1 and Example 3, $\mathcal{A}$ meets this test. To prove this, we have to gather some information about the geometry of this unit ball. We begin with elementary but fundamental results about the ball, and then use the $\lambda$-function of Aron and Lohman [1]. Eventually we shall show that the extreme points found in Theorem 4 are all the extreme points of $\mathbb{B}(A)$. We give the arguments for Example 3. They are easily modified to apply to Example 1.

Theorem 5. Let $X, \mathcal{F}, \mathcal{B}, \mu$ be as in Example 3. Let $\left\{E_{i}\right\}$ be a partition of $[0,1]$ into maximal localizing sets for $\mathcal{B}$. Then any function $f$ in $\mathcal{A}$ with $\sum_{i=1}^{n}\left\|f \chi_{E_{i}}\right\|_{\infty} \leq n$ is a convex combination of at most $2 n$ extreme points in $\mathbb{B}(\mathcal{A})$.

Proof. There exist positive numbers $a_{i}$ with $\sum_{i=1}^{n} \frac{1}{a_{i}}=1$ and $\left\|a_{i} f \chi_{E_{i}}\right\|_{\infty} \leq n$ for each $i$. Mimicking the argument that each complex number of modulus less than one is a convex combination of two points on the unit circle, $\left(a_{i} / n\right) f \chi_{E_{i}}$ is a convex combination of (at most) two unitary elements of $L^{\infty}\left(E_{i}\right)$. Extend each such unitary $u$ to be 0 where it was previously undefined and notice that $\|n u\| \leq 1$. Indeed, each $n u$ is an extreme point of $\mathbb{B}(\mathcal{A})$. Thus $f=\sum_{i=1}^{n} \frac{1}{a_{i}} n\left(\frac{a_{i}}{n} f \chi_{E_{i}}\right)$ is obtained as a convex combination of $2 n$ elements $n u$ with $u$ as in Theorem 4 .

COROLlary 1. In Example 3, every element of the closed ball of radius $\frac{1}{n}$ in $\mathcal{A}$ is a convex combination of at most $2 n$ extreme points.

Proof. The hypothesis $\|f\| \leq \frac{1}{n}$ implies that $\|f\|_{\infty} \leq 1$ and so $\sum_{i=1}^{n}\left\|f \chi_{E_{i}}\right\| \leq n$.

COROLlary 2. In Example 3, every function in $\mathbb{B}(\mathcal{A})$ that is in the range of $\Phi$ is a convex combination of at most $2 n$ extreme points.

Proof. $\Phi(f)=f$ together with $\|f\| \leq 1$ easily implies that $\|f\|_{\infty} \leq 1$ and so the hypothesis of Theorem 5 is satisfied.

We now recall the uniform $\lambda$-property of Aron and Lohman [1, p. 211]. For any normed space $X, x \in \mathbb{B}(X), e$ an extreme point of $\mathbb{B}(X), y \in \mathbb{B}(X), 0<\lambda \leq 1$, and $x=$ $\lambda e+(1-\lambda) y$, say, the triple $(e, y, \lambda)$ is amenable to $x$. In that case, define

$$
\lambda(x)=\sup \{\lambda \in(0,1]: \text { there exists a triple }(e, y, \lambda) \text { amenable to } x\} .
$$

If each $x$ in $\mathbb{B}(X)$ admits an amenable triple, then $X$ has the $\lambda$-property. If, in addition, inf $\{\lambda(x): x \in \mathbb{B}(X)\}>0, X$ has the uniform $\lambda$-property.

The next task is to prove the following result.

TheOREm 6. Let $X, \mathcal{F}, \mathcal{B}, \mu$ be as in Example 3. Then $\mathcal{A}$ has the uniform $\lambda$-property, with $\lambda(a) \geq \frac{1}{n+2}$, for all $a \in \mathbb{B}(\mathcal{A})$.

Proof. Recall that $n$ is fixed in the definition of $\mathcal{A}$. For each $x \in[0,1]$, define a subset $E(x)$ of $[0,1]$ by $E(x)=\left\{x+\frac{k}{n}(\bmod 1): k\right.$ is an integer between 0 and $\left.n-1\right\}$. Define $E=\left\{t: t=\min \left\{y: y \in E(x)\right.\right.$ and $\left.|a(y)|=\max _{z \in E(x)}|a(z)|\right\}$ for some $\left.x \in[0,1]\right\} . E$ is 
clearly Lebesgue measurable, and we wish to show that it is a maximal localizing set for $\mathcal{B}$. Because $E$ contains exactly one element of $E(x)$ for each $x$, every Lebesgue measurable subset of $E$ is the intersection of $E$ with a subset of $\mathcal{B}$; i.e., $E$ is a localizing set for $\mathcal{B}$. Let $\mathcal{F}$ be any Lebesgue measurable set properly containing $E$. $F \cap E^{c} \subset\{x \in$ $[0,1]: F$ contains more than one element of $E(x)\}$. Thus we may assume that the latter set has positive Lebesgue measure. In that case, $F$ is not a localizing set. Thus $E$ is a maximal localizing set.

Now consider an arbitrary $a \in \mathbb{B}(\mathcal{A})$. Define $e$ by

$$
e(x)= \begin{cases}0 & \text { if } x \notin E, \\ n \frac{a(x)}{|a(x)|} & \text { if } x \in E \text { and }|a(x)| \geq \frac{n}{n+2}, \\ n & \text { otherwise. }\end{cases}
$$

By Theorem 4, $e$ is an extreme point of $\mathbb{B}(\mathcal{A})$.

For each $\lambda \in[0,1),(e, y, \lambda)$ is amenable to $a$ if $y=\frac{1}{1-\lambda} a-\frac{\lambda}{1-\lambda} e$ and $\|y\|$ $\leq 1$. Taking the preceding formula as the definition of $y, \lambda(a) \geq \sup \{\lambda:\|a-\lambda e\|$ $\leq 1-\lambda\}$. Observe that $\|a-\lambda e\|=\sup _{x} \frac{1}{n} \sum_{z \in E(x)}|a(z)-\lambda e(z)|$. Let $K$ denote $\sup _{x} \frac{1}{n} \sum_{z \in E(x)}|a(z)-\lambda e(z)|$, and assume that $\lambda \leq \frac{1}{n+2}$.

Case 1. $x \in E$ and $|a(x)| \geq \frac{n}{n+2}$. Here

$$
\begin{aligned}
K & =\frac{1}{n}\left[\left|a(x)-n \lambda \frac{a(x)}{|a(x)|}\right|+\sum_{z \in E(x), z \neq x}|a(z)|\right] \\
& =\frac{1}{n}\left[|| a(x)|-n \lambda|+\sum_{z \in E(x), z \neq x}|a(z)|\right] \\
& \leq\|\Phi(|a|)\|_{\infty}-\lambda=\|a\|-\lambda \leq 1-\lambda .
\end{aligned}
$$

Case 2. $x \in E$ and $|a(x)|<\frac{n}{n+2}$. Here

$$
\begin{aligned}
K & =\frac{1}{n}\left[|a(x)-n \lambda|+\sum_{z \in E(x), z \neq x}|a(z)|\right] \\
& \leq \frac{1}{n}\left[\frac{n}{n+2}+n \lambda+\frac{n(n-1)}{n+2}\right] \\
& \leq 1-\lambda,
\end{aligned}
$$

since $\lambda \leq \frac{1}{n-2}$.

The cases with $x \notin E$ yield $K \leq 1-\lambda$ when $\lambda \leq \frac{1}{n+2}$ by the same arguments because exactly one element of $E(x)$ will be in $E$. Thus $\mathcal{A}$ has the uniform $\lambda$-property with $\lambda(a) \geq \frac{1}{n+2}$.

THEOREM 7. Let $X, \mathcal{F}, \mathcal{B}, \mu$ be as in Example 3. Then every extreme point of $\mathbb{B}(\mathcal{A})$ is one of the type described in Theorem 4.

Proof. Let $a$ be an extreme point of $\mathbb{B}(\mathcal{A})$. In the proof of Theorem 6 we exhibited an extreme point $e$ of the desired type and an element $y$ of $\mathbb{B}(\mathcal{A})$ such that $a$ was a convex combination of $e$ and $y$. The hypothesis that $a$ is extreme implies that $a=e$. 
THEOREM 8. In Example 3, every element of the open unit ball of $\mathcal{A}$ is a convex combination of extreme points of $\mathbb{B}(\mathcal{A})$.

Proof. By Theorem 4 above and Theorem 3.1 of [1], for all $a \in \mathbb{B}(\mathcal{A})$ there exists a sequence of extreme points $e_{k}$ of $\mathbb{B}(\mathcal{A})$ such that

$$
\left\|a-\sum_{k=1}^{n} \lambda(1-\lambda)^{k-1} e_{k}\right\| \leq(1-\lambda)^{n} .
$$

Then

$$
\left\|a-\frac{1}{\sum_{k=1}^{n} \lambda(1-\lambda)^{k-1}} \sum_{k=1}^{n} \lambda(1-\lambda)^{k-1} e_{k}\right\| \leq 2(1-\lambda)^{n},
$$

so that the convex combinations of extreme points are norm dense in $\mathbb{B}(\mathcal{A})$.

However, recall that by Corollary 1 to Theorem 5, every element of the open ball of radius $\frac{1}{n}$ may be expressed exactly as a convex combination of extreme points. It is now simple to verify that if $\|a\|<1$, we may choose $b$ with norm less than 1 such that $b$ is a convex combination of extreme points and $a$ is on the line segment between $b$ and an element norm less than $\frac{1}{n}$. (The idea is to use the fact that $a$ is on the line segment between 0 and $(1+\epsilon) a$ and choose $b$ sufficiently close to $(1+\epsilon) a$.) Thus every element of the open unit ball of $\mathcal{A}$ is a convex combination of extreme points.

We have seen examples where $\mathbb{B}(\mathcal{A})$ has no extreme points and other examples where $\mathbb{B}(\mathcal{A})$ has sufficiently many extreme points for every point of the open unit ball to be a convex combination of extreme points. Of course there are also intermediate cases. For example, let $X=[-1,1] \cup[2,3], \mathcal{F}=\mathcal{L}(X), \mathcal{B}$ the $\sigma$-algebra generated by $\{E: E \subset[-1,1]$ is symmetric about the origin $\} \cup[2,3]$ and $\mu=\mu_{X}$. Then

$$
\Phi(f)(x)= \begin{cases}\frac{1}{2}[f(x)+f(-x)] & \text { if } x \in[-1,1] \\ \int_{2}^{3} f(t) d t & \text { if } x \in[2,3] .\end{cases}
$$

By arguments given earlier, it is clear that, for example, the function $u=2 \chi_{[-1,0]}$ is an extreme point of $\mathbb{B}(\mathcal{A})$. On the other hand, it is equally clear that no function supported on $[2,3]$ is a convex combination of extreme points of the unit ball.

We close then with the following questions for future investigation.

1. If no element of $\mathcal{F}$ is a localizing set for $\mathcal{B}$, can $\mathbb{B}(\mathcal{A})$ have extreme points?

2. If the conditional expectation $\Phi$ satisfies Lambert's condition that for some constant $C,|f| \leq C \Phi(|f|)$ a.e., is every element of the open unit ball of $\mathcal{A}$ a convex combination of extreme points of $\mathbb{B}(\mathcal{A})$ ?

\section{REFERENCES}

1. R. M. Aron and R. H. Lohman, A geometric function determined by extreme points of the unit ball of a normed space, Pacific J. Math. 127 (1987), 209-231.

2. J. T. Campbell, A. Lambert and B. M. Weinstock, Localizing sets and the structure of sigma-algebras, Indiana Univ. Math. J. 47 (1998), 913-938. 
3. J. Daughtry, A. Lambert and B. Weinstock, Operators on $\mathrm{C}^{*}$-algebras induced by conditional expectations. Rocky Mountain J. Math. 25 (1995), 1243-1275.

4. A. Lambert, Localising sets for sigma algebras and related point transformations, Proc. Roy. Soc. Edinburgh Sect. A 118 (1991), 111-118.

5. A. Lambert, $L^{p}$ multipliers and nested sigma-algebras, Operator Theory: Advances and Applications 104 (1998), 147-153.

6. A. Lambert and B. M. Weinstock, A class of operator algebras induced by probabilistic conditional expectations, Michigan Math. J. 40 (1993), 359-376. 